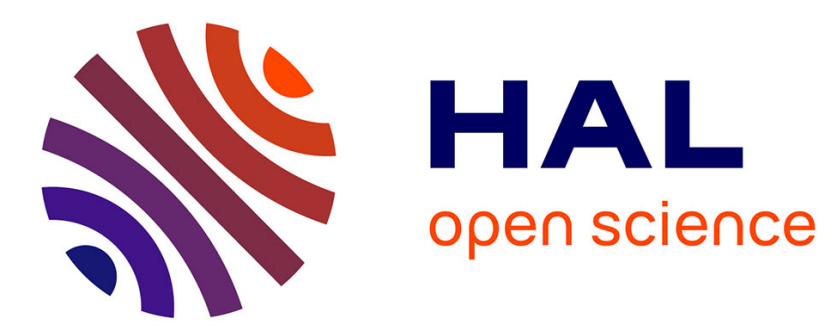

\title{
On the Optimization of a PSP-based CPM Detection
}

\author{
Malek Messai, Karine Amis Cavalec, Frédéric Guilloud
}

\section{To cite this version:}

Malek Messai, Karine Amis Cavalec, Frédéric Guilloud. On the Optimization of a PSP-based CPM Detection. IEEE Transactions on Wireless Communications, 2016, 15 (3), pp.2144 - 2154. 10.1109/TWC.2015.2498932 . hal-01514614

\section{HAL Id: hal-01514614 https://hal.science/hal-01514614}

Submitted on 26 Apr 2017

HAL is a multi-disciplinary open access archive for the deposit and dissemination of scientific research documents, whether they are published or not. The documents may come from teaching and research institutions in France or abroad, or from public or private research centers.
L'archive ouverte pluridisciplinaire HAL, est destinée au dépôt et à la diffusion de documents scientifiques de niveau recherche, publiés ou non, émanant des établissements d'enseignement et de recherche français ou étrangers, des laboratoires publics ou privés. 


\title{
On the Optimization of a PSP-based CPM Detection
}

\author{
Malek MESSAI Student Member, IEEE, Karine AMIS and Frédéric GUILLOUD, Member, IEEE,
}

\begin{abstract}
This paper deals with a reduced-complexity per survivor processing-based CPM demodulation. It relies on a trellis with reduced state number and defined from a rational modulation index possibly different from the transmit modulation index and referred to as virtual receiver modulation index. The virtual receiver modulation index should be chosen so as to achieve a tradeoff between error-rate performance and complexity reduction. The main purpose of this paper is the choice of the virtual receiver modulation index. It gives guidelines to discard the values of the virtual receiver modulation index, that degrade the error-rate performance. Two criteria are used. The first one considers the uncoded CPM case and is based on an approximation of the minimal Euclidean distance. The second one is related to the bit interleaved coded CPM case and resorts to an EXIT chart to analyse the convergence of the iterative receiver.

Index terms: continuous phase modulation, EXIT charts, minimum Euclidean distance, per survivor processing, iterative decoding.
\end{abstract}

\section{INTRODUCTION}

Continuous phase modulation (CPM) [1] is an attractive modulation which combines a constant signal envelope and an excellent bandwidth efficiency [2], [3]. These properties account for its widespread use in digital communication systems. CPM has gained extensive attention since it was developed in 1980s [1]. Recently some results show that CPM is more adequate than linear modulation in power efficient systems including satellite [4], [5], deep-space [6], DVB [7], optical fiber [8], telemetry communication [9], etc. The analysis of communication evolution predicts an increase in the number of connected machines. This is related especially to the regular emergence of surveillance applications/remote control in various fields such as the environment (to anticipate the volcanic eruptions for example), health (for tracking a patient at home since the surgery) or industry (sensor networks, smart meters) [10], [11]. Power and cost efficient digital modulation is required for both environmental and economical reasons, the power consumption in the link should be as low as possible. Continuous phase modulation offers the possibility to use nonlinear cost-effective and power efficient amplifiers. However, in the case of optimal detection, the advantages of CPM are balanced with the complexity at the receiver side. Therefore, receiver complexity reduction techniques are necessary. Due

Manuscript received February 20, 2015; revised July 16 and October 06, 2015; accepted November 04, 2015. The associate editor coordinating the review of this paper and approving it for publication was Song Lingyang.

The paper has been presented in part at the IEEE International Symposium on Turbo Code (ISTC'14), Bremen, Germany, august 2014, and at the 22nd European Signal Processing Conference (EUSIPCO'14), Lisbonne, Portugal, september 2014

The authors are with the Signal and Communication Department of Telecom Bretagne-Institut Telecom, CNRS Lab-STICC (UNR 6285), Brest 29200, France (e-mail:malek.messai, karine.amis, frederic.guilloud@ telecombretagne.eu). to the memory property introduced by the phase continuity [12], optimal detection of CPM signals relies on a maximum likelihood sequence detection (MLSD) approach, which can be implemented using the Viterbi algorithm (VA) [13]. The complexity of the MLSD demodulator highly depends on the number of matched filters and on the number of states in the VA. The Viterbi algorithm is optimal, but has two major drawbacks. First, it can be implemented only when the modulation index is a rational number of the form $\frac{k}{p}$, where $k$ and $p$ are relatively prime positive integers [14], [15]. Otherwise, the number of states is unbounded and the Viterbi receiver has infinite complexity. Secondly, to achieve optimal performance, it requires the exact knowledge of the modulation index. Indeed, whatever the signal to noise ratio, any mismatch on the modulation index can yield errors that accumulate over the whole observation interval. Such a mismatch may have several origins. For example, in analog implementations, the modulation is performed by voltage-controlled oscillators (VCO). In this case, the modulation index is defined by the specifications of electronic components which may vary from one transmitter to another and is hardly exactly the same as specified. Another example is when the modulation index is not specified by a single value but rather by a minimum and a maximum value, like in the Bluetooth standard. It could then be desirable to come up with a receiver having a single structure whatever the modulation index is. Several approaches for reducing the receiver complexity, applied to the coherent detection of CPM signals, have been presented in the literature. The reduction of complexity is generally obtained by decreasing the number of trellis states and/or the number of matched filters (MF). As for only reducing the number of processed trellis states, a reduced-search algorithm performed on the full trellis has been proposed in [16]. In [17] a decision feedback equalizer is used on a smaller trellis. Neither of these methods has any impact on the number of MFs. Svensson and Aulin [18] proposed a detector based on a CPM scheme simpler than the one used at the transmitter. The main idea is to deform the frequency pulse response so as to obtain a shorter frequency pulse response length, thereby reducing the number of trellis states and the number of MFs simultaneously. This is called a mismatched detector, since the internal signal model in the detector is mismatched (different frequency pulses) with respect to the signal produced by the transmitter. Other works are based on the exact decomposition of the CPM signal on an orthonormal basis. The complexity reduction is achieved at the receiver by using a subset of orthonormal basis functions instead of the entire set. In [19], the Gram-Schmidt procedure is applied to obtain the orthonormal basis functions. Other approaches have used sampling functions [20], [21], Walsh functions [22], and regularly spaced sinusoids [19]. Another interesting representation of CPM was introduced by Laurent 
in 1986 [23]. In his paper, Laurent showed that any binary CPM signal can be exactly represented by a linear combination of pulse amplitude modulated (PAM) waveforms. The PAM representation of CPM has received a lot of attention in the literature. It has been exploited by Kaleh to design reduced complexity detectors [24]. These detectors are based on a small subset of pseudo-symbols and pulses yielding a reduction of the MFs number. Therefore, PAM-based detectors achieve a simultaneous reduction in the number of MFs (pulses) and trellis states. The Rimoldi decomposition proposed in [25] gives a concise description of CPM as the concatenation of a continuous-phase encoder (CPE) and a memoryless modulator $(\mathrm{MM})$. This CPM decomposition is of great interest when concatenated with an outer forward error correction (FEC) code. It opens the door to iterative receivers of the concatenated scheme, with near-optimum error rate performance. Different schemes for bit interleaved coded CPM (BIC-CPM) have been proposed [26], [27].

In this paper, we focus on two low-complexity algorithms recently proposed by the authors in [28] and [29], for uncoded and coded coherent CPM signal detection, the CPM modulation index $h$ being not necessarily rational. We first recall the construction of a trellis defined from a rational modulation index $h_{v}$ possibly different from $h$. We then describe a decoding algorithm based on this reduced state-number trellis by modifying the branch metric so as to take into account the difference between $h_{v}$ and $h$. The hard-decision (respectively soft-decision) demodulation is achieved by a modified Viterbi algorithm [28] (respectively modified BCJR algorithm [30]). The modified BCJR algorithm can be used for soft-in soft-out CPM detection in the iterative receiver of a BIC-CPM scheme [29]. The major contribution of this paper deals with the choice of $h_{v}$. We give guidelines to discard the bad values of $h_{v}$, that is to say the values that degrade the error-rate performance. We use two criteria, one based on an approximation of the minimal Euclidean distance and the other based on an EXIT chart analysis [31].

The paper is organized as follows: we first briefly introduce the signal model and the notations in Section II. Then, we describe the low complexity coherent receiver for CPM signals for any modulation index, initially proposed in [28] and [29] in Section III. The virtual reception modulation index choice is studied in Section IV. Finally a conclusion and some perspectives are given in Section V.

\section{Signal Model}

The complex baseband CPM signal is defined as:

$$
s(t, h, \boldsymbol{a})=\sqrt{\frac{E}{T}} e^{j \phi(t, h, \boldsymbol{a})},
$$

where $E$ is the average symbol energy, $T$ is the symbol duration and $\phi(t, h, \boldsymbol{a})$ is the information-bearing phase given by:

$$
\phi(t, h, \boldsymbol{a})=2 \pi h \sum_{i=0}^{\infty} a_{i} q(t-i T) .
$$

$\boldsymbol{a}=\left\{a_{i}\right\}$ denotes the information sequence. The information symbols $a_{i}$ are assumed to be independent and identically distributed and to take values in the $M$-ary alphabet $\mathcal{M}=$ $\{ \pm 1, \pm 3, \ldots, \pm(M-1)\} . q(t)$ is the phase response and it is expressed as:

$$
q(t)=\int_{-\infty}^{t} g(\tau) d \tau
$$

where $g(t)$ is the normalized frequency pulse, defined for $t \in$ $\left[\begin{array}{ll}0, & L T\end{array}\right]$ with $L$ the frequency pulse length. The normalized frequency pulse should satisfy the two following conditions:

$$
\begin{aligned}
g(t) & =g(L T-t), \\
\int_{0}^{t} g(\tau) d \tau & =q(L T)=\frac{1}{2}, \quad t \geq L T .
\end{aligned}
$$

Using the properties of $q(t)$ and $g(t)$ given in Eq. (3) and Eq. (4), the information-bearing phase during the $n$-th time interval, $t \in[n T,(n+1) T], n \in \mathbb{N}$, can be written as:

$$
\begin{aligned}
\phi(t, h, \boldsymbol{a}) & =2 \pi h \sum_{i=0}^{\infty} a_{i} q(t-i T), \\
& =\pi h \sum_{i=0}^{n-L} a_{i}+2 \pi h \sum_{i=n-L+1}^{n} a_{i} q(t-i T), \\
& =\theta_{h, n}+\phi_{h, n}(t) .
\end{aligned}
$$

From Eq. (5) we observe that the modulated signal over the $n$-th time interval depends both on the phase state denoted by $\theta_{h, n}$ and on the $L$ most recent symbols, i.e $\left(a_{n}, a_{n-1}, \ldots, a_{n-L+1}\right)$. For a rational modulation index $h=$ $\frac{l}{p}$, the phase state $\theta_{h, n}$ modulo $2 \pi$ can take only $p$ different values. Therefore, the phase evolution can be described by a finite-state machine, where each state is represented by an $L$-dimensional vector $\left(\theta_{h, n}, a_{n-1}, a_{n-2}, \ldots, a_{n-L+1}\right)$ and where the number of such states is $p M^{L-1}$ or $2 p M^{L-1}$ respectively for $l$ even or odd. The tilted phase obtained via the Rimoldi decomposition [12] yields a number of states equal to $p M^{L-1}$ whatever the parity of $l$. In this paper, the classic phase definition will be considered. Note however that the algorithms and the reasoning proposed in this paper still apply on Rimoldi decomposition-based trellis. We assume that the signal is transmitted over a Gaussian channel. The equivalent baseband received signal, denoted by $r(t)$, is defined as:

$$
r(t)=s(t, h, \boldsymbol{a})+n(t),
$$

where $n(t)$ is a realization of a zero-mean wide sense stationary complex circularly symmetric Gaussian noise, independent of the signal, and with double-sided power spectral density $2 N_{0}$ over the bandwidth of $s(t, h, \boldsymbol{a})$. The Maximum Likelihood sequence estimation (MLSE-detector) aims at maximizing the scalar product between $r(t)$ and all the realizations of $s(t, h, \boldsymbol{a})$. Assuming $N$ transmitted symbols, the MLSE estimation of the information symbols $a_{0}, a_{1}, \ldots, a_{N-1}$ is given by:

$$
\left(\hat{a}_{0}, \hat{a}_{1},, \ldots, \hat{a}_{N-1}\right)=\underset{\boldsymbol{a} \in \mathcal{M}^{N}}{\arg \max } \Re\left[\int_{0}^{N T} r(t) s^{*}(t, h, \boldsymbol{a}) d t\right]_{(7)}
$$

where $\Re(X)$ denotes the real part of $X$. As $\int_{0}^{N T} r(t) s^{*}(t, h, \boldsymbol{a}) d t=\sum_{n=0}^{N-1} \int_{n T}^{(n+1) T} r(t) e^{-j \phi(t, h, \boldsymbol{a})} d t$, 
the computation of all scalar products over $[0, N T]$ is equivalent to the sum of all scalar products over a symbol duration given the previous phase state $\theta_{h, n}$. Let $x_{n}^{j}$ stand for the $j$-th state at the time epoch $n$. We introduce and denote by $\mathrm{BM}_{i, k}^{(n)}$ the branch metric corresponding to the scalar product associated to the transition from state $\boldsymbol{x}_{n}^{k}=\left(\theta_{h, n}^{k}, a_{n-1}^{k}, a_{n-2}^{k}, \ldots, a_{n-L+1}^{k}\right)$ to state $\boldsymbol{x}_{n+1}^{i}=\left(\theta_{h, n+1}^{i}, a_{n}^{i}, a_{n-1}^{i}, \ldots, a_{n-L+2}^{i}\right)$, where $a_{n}^{i}$ is the symbol related to this transition during the $n$-th time interval, $a_{n-\ell}^{k}=a_{n-\ell}^{i}$ and $\theta_{h, n+1}^{i}=\theta_{h, n}^{k}+\pi h a_{n-L+1}^{k}$. $\mathrm{BM}_{i, k}^{(n)}$ is calculated as follows:

$$
\mathrm{BM}_{k, i}^{(n)}=\Re\left\{\int_{n T}^{(n+1) T} r(t) e^{-j \phi_{k, i}(t, h)} d t\right\},
$$

with $\phi_{k, i}(t, h)=\theta_{h, n}^{k}+2 \pi h \sum_{\ell=n-L+1}^{n-1} a_{\ell}^{k} q(t-\ell T)+$ $2 \pi h a_{n}^{i} q(t-n T)$. The Viterbi algorithm is based on the recursive computation of the accumulated metric at state $\boldsymbol{x}_{n+1}^{i}$ and time instant $(n+1) T$ denoted by $\mathrm{CM}_{i}^{(n+1)}$ and defined as:

$\mathrm{CM}_{i}^{(n+1)}=\max _{k}\left(\mathrm{CM}_{k}^{(n)}+\mathrm{BM}_{k, i}^{(n)}\right)$. At the end, the MLSEbased decision is given by the sequence $\hat{\boldsymbol{a}}$ yielding the maximum of $\mathrm{CM}_{i}^{(N)}$ over $i$.

\section{REDUCED-STATE TRELLIS-BASED CPM RECEIVERS}

In this section, the algorithm developed in [28] for any type of CPM is reminded for clarity's sake. The resulting reducedcomplexity receiver relies on the decomposition of $h$ in the form $h=h_{v}+\Delta_{h}$ with $h_{v}=\frac{l_{v}}{p_{v}}$ being a rational number. $h_{v}$ is referred to as a virtual receiver modulation index. The key idea is to use either the Viterbi or the BCJR algorithm with modified branches and state metrics on a trellis designed upon $h_{v}$. It takes into account a phase difference proportional to $\Delta_{h}$ and computed on a Per Survivor Processing basis [32].

\section{A. Design of a Trellis with a Reduced State Number}

Thanks to the decomposition of $h$ in the form $h=h_{v}+$ $\Delta_{h}$, the information-bearing phase given in Eq. (5), can be expressed as a function of $h_{v}$ and $\Delta_{h}$ :

$$
\begin{aligned}
\phi(t, h, \boldsymbol{a})= & \pi h \sum_{i=0}^{n-L} a_{i}+2 \pi h \sum_{i=n-L+1}^{n} a_{i} q(t-i T), \\
= & \pi h_{v} \sum_{i=0}^{n-L} a_{i}+\pi \Delta_{h} \sum_{i=0}^{n-L} a_{i} \\
& +2 \pi h_{v} \sum_{i=n-L+1}^{n} a_{i} q(t-i T) \\
& +2 \pi \Delta_{h} \sum_{i=n-L+1}^{n} a_{i} q(t-i T), \\
= & \underbrace{\theta_{h_{v}, n}+\phi_{h_{v}, n}(t)}_{\phi\left(t, h_{v}, \boldsymbol{a}\right)} \\
& +\underbrace{\theta_{\phi\left(t, \Delta_{h}, \boldsymbol{a}\right)}}_{\Delta \theta_{n}^{i}+2 \pi \Delta_{h} \sum_{i=n-L+1}^{n}} a_{i} q(t-i T)
\end{aligned}
$$

with $\theta_{h_{v}, n}=\pi h_{v} \sum_{i=0}^{n-L} a_{i}, \quad \phi_{h_{v}, n}(t)=$ $2 \pi h_{v} \sum_{i=n-L+1}^{n} a_{i} q(t-i T)$ and $\Delta \theta_{n}^{i}=\pi \Delta_{h} \sum_{i=0}^{n-L} a_{i}$.

The first two terms in Eq. (9) are tracked by the Viterbi algorithm and the third term is the resulting phase difference which is accumulated at every symbol. This accumulation is calculated using the PSP technique by associating to each state an additional parameter $\Delta \theta_{n}^{i}$. The last term of Eq. (9) is calculated at the output of the matched filter. We can write the following relation:

$$
\begin{aligned}
\theta_{h}^{i} & =\Delta \theta_{n}^{i}+i \frac{l_{v}}{p_{v}} \pi, \quad i=\left\{0,1,2, \ldots, p_{v}-1\right\}, \\
& =\Delta \theta_{n}^{i}+\theta_{h_{v}}^{i} .
\end{aligned}
$$

The modified branch metric is equal to the scalar product which is calculated as follows:

$\mathrm{BM}_{k, i}^{\text {modified }}=\Re\left(e^{-j \Delta \theta_{n}^{k}} \int_{n T}^{(n+1) T} r(t) e^{-j \phi_{k, i}\left(t, h_{v}\right)} \cdot e^{-j \psi_{k, i}\left(t, \Delta_{h}\right)} d t\right)$.

where

$$
\begin{aligned}
\phi_{k, i}\left(t, h_{v}\right)= & \theta_{h_{v}, n}^{k}+2 \pi h_{v} \sum_{\ell=n-L+1}^{n-1} a_{\ell}^{k} q(t-\ell T) \\
& +2 \pi h_{v} a_{n}^{i} q(t-n T) \\
\psi_{k, i}\left(t, \Delta_{h}\right)= & 2 \pi \Delta_{h} \sum_{\ell=n-L+1}^{n-1} a_{\ell}^{k} q(t-\ell T) \\
& +2 \pi \Delta_{h} a_{n}^{i} q(t-n T)
\end{aligned}
$$

1) PSP-based Modified Viterbi Algorithm: The only additional task that needs to be performed on the Viterbi algorithm is the PSP update of the phase difference for each state, $\Delta \theta_{n}^{i}$. The update equation is given by:

$$
\Delta \theta_{n+1}^{i}=\Delta \theta_{n}^{k^{*}}+a_{\left(k^{*}, i\right)} \pi \Delta_{h},
$$

where $k^{*}$ is selected from the $M$ previous states of $\boldsymbol{x}_{(n+1)}^{i}$, as the index of the maximum cumulative metric and $a_{\left(k^{*}, i\right)}$ is the last component of the state vector $\boldsymbol{x}_{n}^{k^{*}}$. We apply Eq. (11) to compute the scalar product between the received signal and the signal corresponding to the transition $\boldsymbol{x}_{n}^{k} \rightarrow \boldsymbol{x}_{n+1}^{i}$ used in the modified Viterbi algorithm:

$$
\begin{aligned}
& \left(\theta_{h_{v}}^{k}\left(\Delta \theta_{n}^{k}\right), a_{n-1}^{k}, a_{n-2}^{k}, \ldots, a_{n-L+1}^{k}\right) \stackrel{a_{n}}{\longrightarrow} \\
& \quad\left(\theta_{h_{v}}^{i}\left(\Delta \theta_{n+1}^{i}\right), a_{n}^{i}, a_{n-1}^{i},, \ldots, a_{n-L+2}^{i},\right),
\end{aligned}
$$

with $a_{n}^{i}=a_{n}, a_{n-1}^{i}=a_{n-1}^{k}, \cdots, a_{n-L+2}^{i}=a_{n-L+2}^{k}$.

2) PSP-based Modified BCJR Algorithm: The optimal coherent soft output detection which minimizes the symbol error probability is achieved by maximum a posteriori (MAP) symbol detection of the information symbol $a_{n}$. The corresponding symbol-by-symbol MAP detector maximizes the a posteriori probability (APP) $p\left(a_{n} \mid r(t)\right)$ :

$$
\hat{a}_{n}=\underset{a \in \mathcal{M}}{\arg \max } p\left(a_{n}=a \mid r(t)\right) .
$$

In the modified BCJR algorithm, we first carry out the forward recursion $\alpha_{n}$, where the branch metric between states $\boldsymbol{x}_{n}^{k}$ and $\boldsymbol{x}_{n+1}^{i}$ is modified as follows:

$$
\gamma_{n}\left(\boldsymbol{x}_{n}^{k}, \boldsymbol{x}_{n+1}^{i}\right) \propto \exp \left(\frac{\mathrm{BM}_{k, i}^{\text {modified }}}{2 N_{0}}\right) p\left(\boldsymbol{x}_{n}^{k} \mid \boldsymbol{x}_{n+1}^{i}\right)
$$


where the term $\Delta \theta_{n+1}^{i}$ is calculated recursively according to Eq. (13), with index $k^{*}$ defined as $k^{*}=$ arg $\max _{k}\left\{\alpha_{n}\left(\boldsymbol{x}_{n}^{k}\right) \gamma_{n}\left(\boldsymbol{x}_{n}^{k}, \boldsymbol{x}_{n+1}^{i}\right)\right\}$, and $a_{\left(k^{*}, i\right)}$ defined as the last component of the state vector $\boldsymbol{x}_{n}^{k^{*}}$. Here, it is important to mention the main difference between the original BCJR algorithm for which the forward and backward recursions are calculated in parallel: in the trellis with a reduced number of states, the backward recursion is carried out once the forward recursion is over. It is thus necessary to keep track of the survivor associated to each transition at each epoch in the forward recursion of $\alpha_{n}\left(\boldsymbol{x}_{n}^{k}\right)$. Then, these survivors are used in the backward recursion of $\beta_{n}\left(\boldsymbol{x}_{n}^{k}\right)$. It means that the value of $\gamma_{n}\left(\boldsymbol{x}_{n}^{k}, \boldsymbol{x}_{n+1}^{i}\right)$ will be saved during the forward recursion to be used in the backward recursion. In fact, the PSP approach is only incorporated in the forward recursion. The reasons for that are twofold: first, the accumulated phase $\Delta \theta_{N}$ associated to last symbol period is unknown, whereas the accumulated phase $\Delta \theta_{0}$ is known and equals zero; second, since the branch metric depends on the accumulated phase state (computed by the PSP process), using the PSP in both the forward and the backward recursions would produce different branch metrics for the same transition, which is not acceptable.

The proposed algorithm is summarized below:

PSP-based modified BCJR algorithm

- Step 1: Initialization $(n=0)$

$\Delta \theta_{0}^{k}=0$, for all states $\boldsymbol{x}_{0}^{k}$,

$\alpha_{0}\left(\boldsymbol{x}^{0}\right)=1, \alpha_{0}\left(\boldsymbol{x}^{k}\right)=0, k \neq 0$.

- Step 2: Forward Recursion $n: 0 \rightarrow N-1$

Calculate for all possible transitions $\boldsymbol{x}_{n}^{k} \rightarrow \boldsymbol{x}_{n+1}^{i}$ in the reduced state number trellis, the branch metrics $\gamma_{n}\left(\boldsymbol{x}_{n}^{k}, \boldsymbol{x}_{n+1}^{i}\right)$ according to Eq. (15). Then, for each $\boldsymbol{x}_{n+1}^{i}$, update $\Delta \theta_{n+1}^{i}$ according to Eq. (13).

- Step 3 : Backward Recursion $n: N \rightarrow 0$

Initialize $\beta_{N}\left(\boldsymbol{x}_{N}^{k}\right)=\frac{1}{N_{s}} \forall k$, where $N_{s}$ denotes the number of states.

Update backward-accumulated metric $\beta_{n}$ by using the branch metrics calculated in the forward step.

\section{B. Iterative Soft Detection for Bit Interleaved Coded CPM}

The serially concatenated CPM signals with iterative decoding were first studied in [25]. The combination of continuous phase modulation (CPM) and bit interleaved coded modulation (BICM) is referred to as BIC-CPM. The PSP-based modified BCJR algorithm has been applied in the iterative receiver by the authors in [29]. The BIC-CPM system is described in Fig. 1. The transmitted bits, $\boldsymbol{b}=\left\{b_{n}\right\}$, are encoded using a convolutional channel encoder. Then, the coded bits $c=\left\{c_{n}\right\}$ are interleaved and mapped to an $M$-ary alphabet $\{ \pm 1, \pm 3, \ldots, \pm(M-1)\}$ to define a symbol sequence denoted by $\boldsymbol{a}=\left\{a_{n}\right\}$.

At each iteration, the proposed soft CPM demodulator computes extrinsic LLRs $\Lambda_{\mathrm{dem}}^{\text {ext }}$ from the channel observations $r(t)$ and a priori LLRs $L_{A}$ which are then passed through the bit interleaver, to become a priori information for the decoder. The latter feeds back in turn extrinsic LLRs $\Lambda_{\mathrm{dec}}^{\mathrm{ext}}$

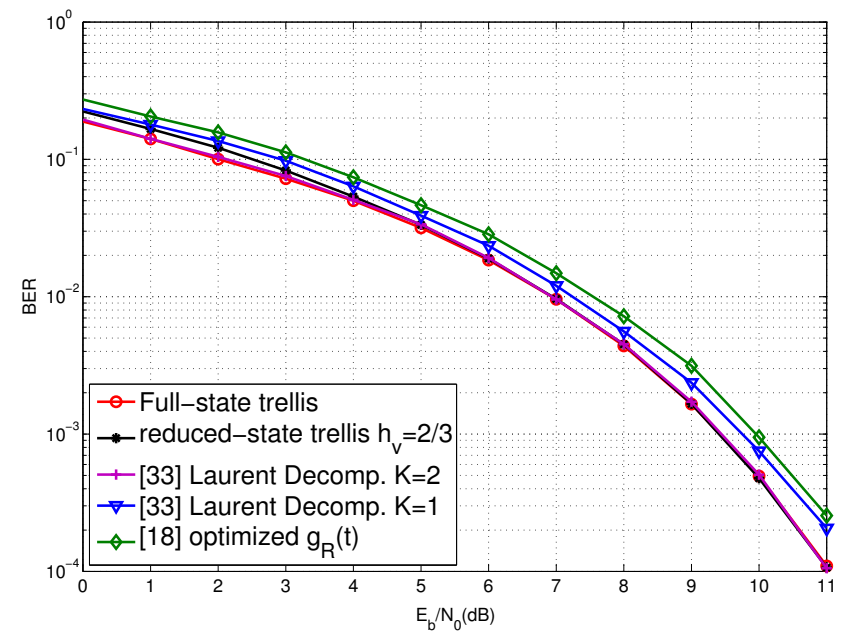

Fig. 2: BER performance comparison for binary 3RC modulation with $h=\frac{3}{8}$.

which are deinterleaved to become new a priori information for the proposed soft CPM demodulator.

\section{Simulations}

1) Comparison with Existing Reduced Complexity Receivers: The proposed receiver is compared with two algorithms described in [18] and [33]. The key idea of [18] is to use a receiver with a modified frequency pulse having a shorter length compared to the frequency pulse used at the transmitter. For example, the binary $3 R C$ transmitted signal is demodulated using a trellis of the binary 2REC modulation. In [33], the Laurent decomposition into a superposition of PAM waveforms is considered with $K$ principal components. Hence, both [18] and [33] consider a signal space at the receiver side which is different from the transmitter side. Note that this is not the case in the proposed receiver where the signal space is unchanged.

For this comparison, we considered the modulation format used in [18], namely a binary 3RC modulation with $h$ set to $\frac{3}{8}$. The phase follows the classical phase definition for CPM, as opposed to the titled phase introduced by Rimoldi. The BER performance is illustrated in Fig. 2, and a comparison of the complexities in terms of number of states is given in Table I.

Compared to the full-state receiver, the proposed reducedstate receiver based upon $h_{v}=\frac{2}{3}$, has 12 states instead of 64 and allows to have a negligible loss for low $E_{b} / N_{0}$ and optimum performance from $E_{b} / N_{0}=4 \mathrm{~dB}$. However, the algorithm proposed in [18] based on a trellis of 32 states, performs $0.6 \mathrm{~dB}$ away from the optimum performance at BER $=10^{-2}$. For the receiver proposed in [18], the approximation of the signal by its two principal components leads to achieve optimal demodulation for each $E_{b} / N_{0}$ with a trellis of 32 states. The approximation of the signal by only its principal component leads to a degradation of $0.3 \mathrm{~dB}$ at $\mathrm{BER}=10^{-2}$ with a trellis of 16 states.

2) Influence of the choice of the virtual receiver modulation index: This section provides numerical results to assess the 


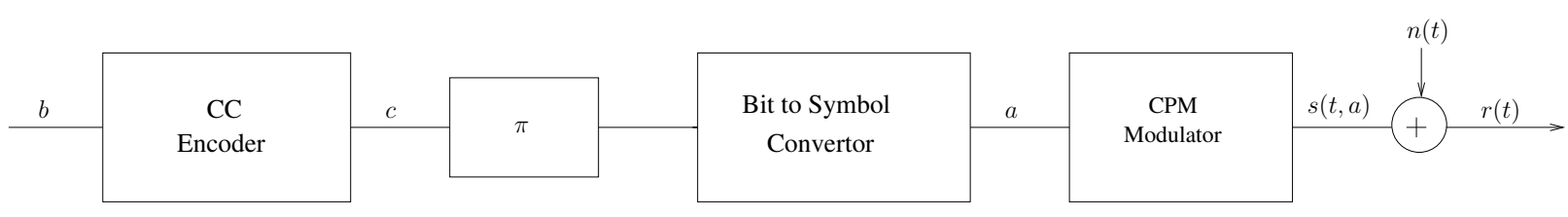

(a) Transmitter.

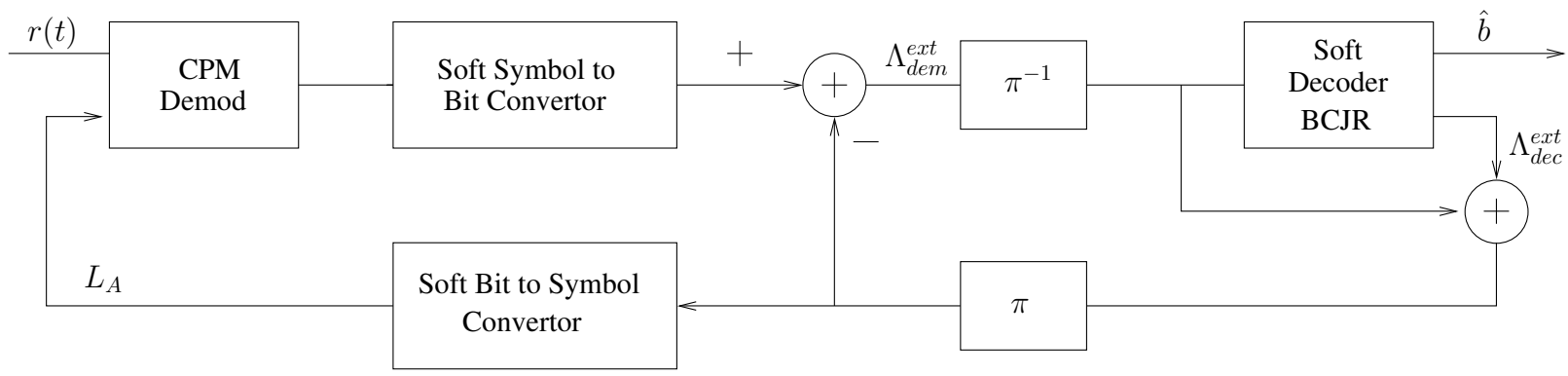

(b) Receiver.

Fig. 1: BIC-CPM system

TABLE I: Complexity reduction vs performance degradation for Binary 3RC modulation, $h=3 / 8$

\begin{tabular}{|l|l|l|l|}
\hline & $\begin{array}{l}\text { Number } \\
\text { of states }\end{array}$ & $\begin{array}{l}\text { Reduction } \\
\text { percentage }\end{array}$ & $\begin{array}{l}\text { Loss }(\mathrm{dB}) \text { at } \\
\text { BER }=10^{-2}\end{array}$ \\
\hline Full-state receiver & 64 & - & - \\
\hline Proposed receiver $\left(h_{v}=2 / 3\right)$ & 12 & $81.25 \%$ & 0 \\
\hline$[18]$ & 32 & $50 \%$ & 0.6 \\
\hline$[33], K=2$ & 32 & $50 \%$ & 0 \\
\hline$[33], K=1$ & 16 & $75 \%$ & 0.3 \\
\hline
\end{tabular}

performance of the studied algorithms with reduced state number on an AWGN channel for both uncoded CPM and BIC-CPM cases. They also illustrate the need for optimization developed in Section IV. In the simulations of the BICCPM, we use a binary convolutional code defined by its polynomials $(7,5)$ in octal and a pseudo-random interleaver. The interleaver length (equal also to the binary coded sequence length), denoted by $K$, takes on its values in $\{4096,20000\}$. We consider a Quaternary and partial response CPM with a raised-cosine frequency pulse of length $L=2$, referred to as Quaternary 2RC CPM in the remainder of the paper. This modulation format is often used in the literature (see for example [34], [35] and [33]). The transmission modulation index is assumed to be rational to enable a comparison with the optimum CPM demodulation.

In the figure legends, uncoded refers to the uncoded scheme, where hard Viterbi-based detection is carried out; coded refers to the BIC-CPM with soft BCJR-based detection involved in the iterative decoding process; $h_{v}=h$ means an optimum CPM demodulation algorithm (either Viterbi or BCJR), working on the trellis with full state number, whereas $h_{v} \neq h$ means a CPM demodulation with the help of the PSP-based modified algorithm (either Viterbi or BCJR), working on a trellis with reduced state number. In the text, we refer to the receiver based on a trellis with the full state number (respectively the receiver based on a trellis with a reduced state number) as full-state receiver (respectively reduced-state receiver).

We consider two reduced-state receivers. The first designed

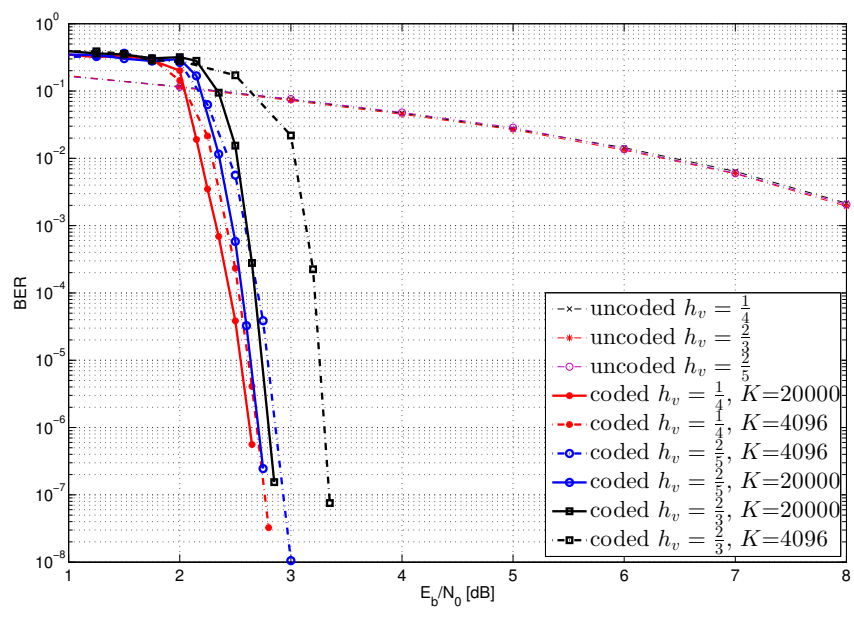

Fig. 3: BER performance of the reduced-state receiver for a Quaternary raised cosine CPM with $L=2$ and $h=\frac{1}{4}$. BIC-CPM: CC(7,5), pseudo-random interleaver and $K \in$ $\{4096,20000\}$.

with $h_{v}=\frac{2}{5}$ has a 20 -state trellis, while the second designed with $h_{v}=\frac{2}{3}$ has a 12-state trellis. The question to be answered is: should we always choose the $h_{v}$ yielding the lowest number of states whatever $h$ ? Partial answer is illustrated by the simulation results given below whereas Section IV will provide a deeper analysis. In Fig. 3 (respectively Fig. 4), we have plotted the BER curve for both uncoded CPM and BICCPM cases, when the transmission modulation index is set to $h=\frac{1}{4}$ (respectively $h=\frac{2}{5}$ ).

Let us first focus on the uncoded case. We observe that for $h=\frac{1}{4}$, both reduced-state receivers achieve the same performance as the full-state receiver. For $h=\frac{2}{5}$, the receiver with $h_{v}=\frac{2}{5}$ becomes the reference full-state receiver and it performs $0.75 \mathrm{~dB}$ better than the reduced-state receiver.

Let us now consider the BIC-CPM case and analyze the 


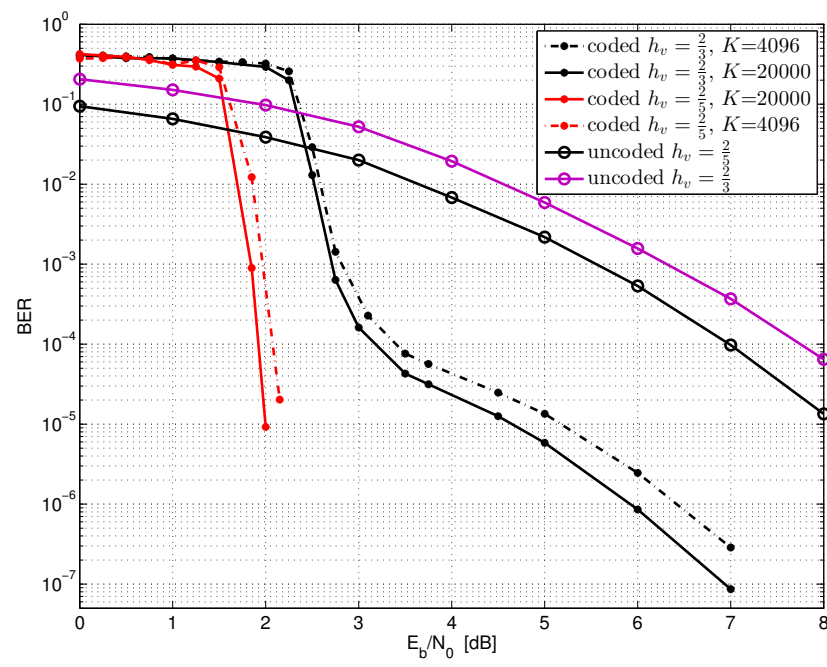

Fig. 4: BER performance of the reduced-state receiver for a Quaternary raised cosine CPM with $L=2$ and $h=\frac{2}{5}$. BIC-CPM: $\mathrm{CC}(7,5)$, pseudo-random interleaver and $K \in$ $\{4096,20000\}$.

receiver convergence depending on $h_{v}$ and $K$. For the BICCPM with $h=\frac{2}{5}$ (cf. Fig. 4), the BER performance of the reduced-receiver is significantly poor compared to the fullsate receiver performance for both interleaver sizes, which was predictable from the uncoded case observation. The SNR gap is of roughly $1 \mathrm{~dB}$ as long as BER $<10^{-3}$ and it keeps increasing below $\mathrm{BER}=10^{-3}$ due to a change of slope beyond around $3 \mathrm{~dB}$. This change of slope is a typical BER shape for iterative receivers. There is first a slow decrease of the BER: the initial BER is too high to enable the iterative process to converge. As soon as the iterative process gain is engaged, the BER decreases quickly with the SNR (the cliff effect): this behaviour can be analysed by computing the iterative transfer of extrinsic information (EXIT charts). Then, from a given SNR value, one observes a change of slope (called the error floor), which corresponds to the convergence of the receiver towards the lower union bound. The union bound depends on the minimum distance of the BIC-CPM scheme and the error floor is all the lower as the minimum distance is high, where the minimum distance is the minimal Euclidean distance between two different coded and modulated sequences.

In the case of the BIC-CPM with $h=\frac{1}{4}$ and $K=4096$ (cf. Fig. 3), the convergence of the full-state receiver occurs after 8 iterations. Comparatively, the reduced-state receiver with $h_{v}=\frac{2}{5}$ (respectively $h_{v}=\frac{2}{3}$ ) converges to its steady state after 15 iterations (respectively 20 iterations) with a loss of $0.2 \mathrm{~dB}$ (respectively $0.65 \mathrm{~dB}$ ) at $\mathrm{BER}=10^{-5}$. In the case of $K=20000$, the convergence of the full-state receiver is achieved after 10 iterations. The reduced-state receiver used with $h_{v}=\frac{2}{5}$ (respectively $h_{v}=\frac{2}{3}$ ) performs close to the fullstate receiver with a slight loss of $0.1 \mathrm{~dB}$ (respectively 0.2 $\mathrm{dB})$ at $\mathrm{BER}=10^{-5}$ and with convergence occurring after 14 iterations (respectively 20 iterations). We deduce that in that particular case, $h_{v}=\frac{2}{5}$ (respectively $h_{v}=\frac{2}{3}$ ) achieves a good tradeoff between error rate and complexity (iteration number required to converge and trellis state number) for medium codeword lengths (respectively high codeword lengths).

3) Conclusion: The proposed receiver has a lower number of states compared to the MLSE receiver, and compared to popular state reduction techniques from the state of the art. The state reduction results from the suppression of parallel paths in the full-state trellis. Two distinct symbol sequences $\boldsymbol{a}$ and $\boldsymbol{b}$ correspond to parallel paths in the full-state trellis from $t=$ $n T$ if their respective states $x_{h, n}^{(a)}=\left(\theta_{h, n}^{(a)}, a_{n-1}, \ldots, a_{n-L+1}\right)$ and $x_{h, n}^{(b)}=\left(\theta_{h, n}^{(b)}, b_{n-1}, \ldots, b_{n-L+1}\right)$ satisfy $\theta_{h, n}^{(a)} \neq \theta_{h, n}^{(b)}$ and $a_{i}=b_{i}$ for $i \geq n-L+1$. These two parallel paths in the fullstate trellis can superimpose in the reduced-state trellis (i.e. the path with the lowest metric is suppressed), if the accumulated phase state of the path $\boldsymbol{a}, \Delta \theta_{n}^{(a)}=\pi \Delta_{h} \sum_{i=0}^{n-L} a_{i}$ (resp. of the path $\boldsymbol{b}, \Delta \theta_{n}^{(b)}=\pi \Delta_{h} \sum_{i=0}^{n-L} b_{i}$ ) satisfy:

$$
\begin{aligned}
& \theta_{h, n}^{(a)}=\left[\theta_{h_{v}, n}+\Delta \theta_{n}^{(a)}\right]_{2 \pi} \\
& \theta_{h, n}^{(b)}=\left[\theta_{h_{v}, n}+\Delta \theta_{n}^{(b)}\right]_{2 \pi}
\end{aligned}
$$

Since parallel paths correspond to the same transmitted symbols but may have different metrics, the suppression of parallel paths has more impact on the symbol-by-symbol decoder than on the sequence decoder.

We can also conclude that $h_{v}$ shall not always be chosen to minimize the trellis state number. Depending on $h$, some $h_{v}$ values may be discarded. Values of $h_{v}$ discarded for the uncoded CPM receiver should also be discarded for the BICCPM reduced-state receiver. However, as observed hereinbefore, some values of $h_{v}$ suitable for the uncoded CPM reducedstate receiver should be discarded for the BIC-CPM reducedstate receiver. In Section IV, rules to help discarding bad $h_{v}$ values are derived.

\section{Choice of the Virtual Receiver Modulation INDEX}

We have seen in the previous section, that the error rate performance of the receiver based on the trellis with reduced state number may be more or less affected by the chosen virtual receiver modulation index $h_{v}$. If two different receiver modulation indices yield the same performance of the reducedstate receiver for the uncoded CPM scheme, this is not necessary the case for the BIC-CPM. Hence, the objective of this section is to study the choice of the virtual receiver modulation index in the cases of both uncoded and coded CPM, and to derive rules to skip some $h_{v}$ values that would lower the error rate performance. All the reasoning presented in this section is performed without loss of generality on the classical phase definition of CPM, and thus could also be straightforwardly applied using the tilted phase of the Rimoldi decomposition.

\section{A. Uncoded CPM Case: Minimum Distance Analysis}

In this section, we focus on the choice of $h_{v}$ to build the trellis with reduced state number in the case of uncoded CPM. More precisely, we derive a rule to discard $h_{v}$ values that 
will surely yield degradations of the error rate performance compared to the optimum performance. Since an analytical expression of the error probability achieved by the MLSE demodulation cannot be obtained, a criterion based on the minimum Euclidean distance is usually used to evaluate the performance of trellis-based CPM demodulation [1]. In the following our reasoning to discard any values of $h_{v}$ is based on an approximation of an upper bound of the minimum Euclidean distance. This section is organized as follows: we first recall some materials of performance analysis based on the minimum Euclidean distance [1] and we underline how to adapt the analysis to the proposed reduced-state receiver. Then we apply this analysis to define rules for discarding values of $h_{v}$ and illustrate the method through simulations.

1) Minimum Euclidean Distance Analysis [1]: The MLSE detection consists in finding the CPM sequence located at the minimum Euclidean distance denoted $d_{\min }$ from the received sequence. When an error occurs at high signal to noise ratio, it corresponds to a decided CPM sequence located at minimum Euclidean distance from the true CPM sequence. For high $E_{b} / N_{0}$, the bit error probability $P_{e}$ under MLSE detection is well approximated by

$$
P_{e} \approx \mathrm{Q}\left(\sqrt{\frac{E_{b}}{N_{0}} d_{\text {min }}^{2}}\right) .
$$

where $\mathrm{Q}(*)$ is the error function defined by

$$
\mathrm{Q}(x)=\int_{x}^{+\infty} \frac{1}{\sqrt{2 \pi}} e^{-u^{2} / 2} d u
$$

Thus the error rate will be all the lower as the minimum Euclidean distance is high. The minimum normalized squared Euclidean distance $d_{\min }^{2}$ is defined as:

$$
d_{\min }^{2}=\min _{\substack{\boldsymbol{a}, \boldsymbol{b} \\ a_{0} \neq b_{0}}}\left(d^{2}(\boldsymbol{a}, \boldsymbol{b})\right)
$$

where $d^{2}(\boldsymbol{a}, \boldsymbol{b})$ is the Euclidean distance between the CPM signals corresponding to the information symbol sequences $\boldsymbol{a}$ and $\boldsymbol{b}$. This Euclidean distance is calculated as:

$$
d^{2}(\boldsymbol{a}, \boldsymbol{b})=\frac{1}{2 E_{b}} \int_{0}^{N T}|s(t, h, \boldsymbol{a})-s(t, h, \boldsymbol{b})|^{2} d t
$$

where $N$ is the number of information symbols. In fact, $d^{2}(\boldsymbol{a}, \boldsymbol{b})$ depends on the difference sequence defined as $\gamma=$ $\boldsymbol{a}-\boldsymbol{b}$ rather than on the individual data sequences themselves, and so Eq. (20) can also be rewritten as [1]:

$$
d^{2}(\boldsymbol{a}, \boldsymbol{b})=d^{2}(\gamma)=\log _{2}(M) \frac{1}{T} \int_{0}^{N T}[1-\cos (\phi(t, h, \gamma))] d t,
$$

where $\phi(t, h, \gamma)$ is the phase trajectory of the difference sequence $\gamma$, and where a notation facility has been introduced by defining $d^{2}(\gamma)$ as $d^{2}(\boldsymbol{a}, \boldsymbol{b})$. Using the decomposition of the modulation index $h=h_{v}+\Delta_{h}$, Eq. (21) can be rewritten as:

$$
\begin{aligned}
& d^{2}(\gamma)=\frac{\log _{2}(M)}{T} \int_{0}^{N T}(1-\cos (\underbrace{\phi\left(t, \Delta_{h}, \gamma\right)}_{(T 1): \text { PSP process }} \\
&+\underbrace{\phi\left(t, h_{v}, \gamma\right)}_{(T 2): \text { reduced trellis }})) d t
\end{aligned}
$$

where the first term (T1) is computed using the PSP technique and where the second term (T2) is tracked by the reduced-state trellis.

The minimization in Eq. (19) is infeasible since the number of difference sequences $\gamma$ satisfying $\gamma_{0} \neq 0$ grows exponentially with $N$ and $M: \operatorname{card}\left(\left\{\gamma \mid \gamma_{0} \neq 0\right\}\right)=(M-1) \times M^{N-1}$. The term inside the integral in Eq. (21) is positive, so $d^{2}(\gamma)$ increases with $N$. This suggests that the difference sequence $\gamma$ corresponding to the minimum distance should have a short duration, that is to say the time interval over which $\gamma$ differs from the all-zero sequence should be limited, thus reducing the integration interval in Eq. (21). Hence, an upper bound denoted by $d_{B}$ on the minimum distance $d_{\min }$ can be found by using only the difference sequences of short duration: the upper bound is then defined on a particular subset of difference sequences denoted by $\Gamma_{f}$. This can be summarized as:

$$
\underbrace{\min _{\gamma, \gamma_{0} \neq 0}\left\{d^{2}(\gamma)\right\}}_{d_{\min }^{2}} \leq \underbrace{\min _{\gamma \in \Gamma_{f}}\left\{d^{2}(\gamma)\right\}}_{d_{B}^{2}}
$$

The set of the difference sequences having a short duration $\Gamma_{f}$ is evaluated thanks to the so-called phase tree built upon $h_{v}$. This tree is formed by the set of phase trajectories having a common start phase, say zero, at time $t=0$. We assume that the data symbols for all the phase trajectories in the tree before this time are equal. The phase trajectories do not coincide over the first symbol interval $\left(\gamma_{0} \neq 0\right)$. However, when going further into the tree, it is always possible to find a pair of phase trajectories which coincide (modulo $2 \pi$ ) at a specific time. This instant is referred to as a merger. Let $\tau$ denote the instant where the two phase trajectories merge. The calculation of the Euclidean distance between both signals can be reduced to the interval $[0, \tau]$. The mergers are easily identified in the phase difference tree, since they correspond to a phase difference trajectory which is identically equal to zero for all $t \geq \tau$. The mergers can be classified by sorting the values of $\tau$ in ascending order. The first order mergers correspond to the smallest values of $\tau$, and the upper bound $d_{B}^{2}$ defined in Eq. (23) can be found by using only the difference sequences corresponding to the first order mergers. The more orders, the tighter the bound.

We denote by $t_{k}$ a $k$-th merger and we assume $t_{k}=m T$. Let $\boldsymbol{a}$ and $\boldsymbol{b}$ be one pair of sequences with phase trajectories merging at time $t_{k}$. Then $\gamma_{i}=a_{i}-b_{i}$ takes on values in $\mathcal{A}=\{0, \pm 2, \pm 4, \ldots, \pm 2(M-1)\}$. The difference phase reads

$$
\phi\left(t, h_{v}, \gamma\right)=2 \pi h_{v} \sum_{i=-\infty}^{m-1} \gamma_{i} q(t-i T)
$$

with $\gamma_{i} \in \mathcal{A}$ for $i=0,1, \ldots, m-1$ and $\gamma_{i}=0$ for $i<0$.

According to Eq. (22), $d(\boldsymbol{a}, \boldsymbol{b})^{2}$ is a function of $\cos \left(\phi\left(t, h_{v}, \gamma\right)\right)$, which is an even function. From Eq. (24), we observe that each sequence $\gamma$ has its opposite in the set of phase difference sequences. Thus, for the calculation of the upper bound of the minimum distance, it is sufficient to consider the phase difference tree, using the difference sequences $\gamma=\left(\gamma_{0}, \ldots, \gamma_{N-1}\right)$ taken in $\Gamma_{m}$ defined as:

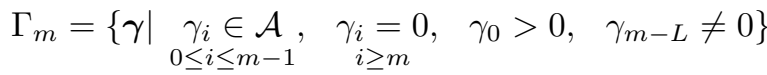


Assuming $\gamma \in \Gamma_{m}$, the two phase trajectories merge at $t_{k}$ and coincide beyond if the following condition is satisfied:

$$
\begin{array}{r}
\exists \ell \in \mathbb{Z}, \phi\left(t, h_{v}, \gamma\right)=2 \ell \pi, t \geq t_{k} \\
\Leftrightarrow \exists \ell \in \mathbb{Z}, \quad 2 \pi h_{v} \sum_{i=0}^{m} \gamma_{i} q(t-i T)=2 \ell \pi, t \geq t_{k} \\
\Leftrightarrow \exists \ell \in \mathbb{Z}, \pi h_{v} \sum_{i=0}^{m-L} \gamma_{i}+2 \pi h_{v} \sum_{i=m-L+1}^{m} \gamma_{i} q(t-i T)=2 \ell \pi,
\end{array}
$$

Let $\boldsymbol{b}$ and $\hat{\boldsymbol{b}}$ be the pair of sequences yielding a phase difference trajectory corresponding to a $k$-th merger. The paths merge at $t_{k}=m T$ at a state $x_{m}^{j}=\left(\theta_{h_{v}, m}^{j}\left(\Delta \theta_{m+1}^{j}\right), a_{m}^{j}, a_{m-1}^{j}, \ldots, a_{m-L+1}^{j}\right)$. It means that $\left(b_{m}, b_{m-1}, \ldots, b_{m-L+1}\right)=\left(a_{m}^{j}, a_{m-1}^{j}, \ldots, a_{m-L+1}^{j}\right)$ and that $\left(\hat{b}_{m}, \hat{b}_{m-1}, \ldots, \hat{b}_{m-L+1}\right)=\left(a_{m}^{j}, a_{m-1}^{j}, \ldots, a_{m-L+1}^{j}\right)$, and thus $\left(b_{m}, b_{m-1}, \ldots, b_{m-L+1}\right)=\left(\hat{b}_{m}, \hat{b}_{m-1}, \ldots, \hat{b}_{m-L+1}\right)$, that is to say $\gamma_{i}=0, i=\{m-L+1, \ldots, m\}$ : so the time dependent part of Eq. (26) is set to zero, yielding:

$$
\text { Eq. (26) } \Leftrightarrow \exists \ell \in \mathbb{N}, \pi h_{v} \sum_{i=0}^{m-L} \gamma_{i}=2 \ell \pi \text {. }
$$

Thus, the difference sequence of a pair of sequences whose phase trajectories separate at $t=0\left(\gamma_{0}>0\right)$ and merge at $t_{k}$, always verifies

$$
\left\{\begin{array}{l}
\exists \ell \in \mathbb{N}, h_{v} \sum_{i=0}^{m-L} \gamma_{i}=2 \ell \\
\gamma_{0}>0
\end{array}\right.
$$

From [1] (Chapter 3), in the case of the MLSE (full-state trellis corresponding to $h_{v}=h$ ), the upper bound on the minimal distance $d_{B}$ is obtained using the difference sequences that are common to all the values of the modulation index i.e when $\ell=0$. The upper bound $d_{B}$ is thus computed for the MLSE considering all sequences satisfying

$$
\sum_{i=0}^{m-L} \gamma_{i}=0, \quad \gamma \in \Gamma_{m}
$$

The proposed algorithm working on the reduced state-number trellis suppresses more candidate paths (due to a higher merging path number per state) compared to the Viterbi algorithm working on the original trellis, yielding a possible smaller minimum Euclidean distance, and thus performance degradation. The virtual modulation index $h_{v}$ will be chosen so that the $k$-th mergers in the trellis with reduced state number are higher than the $k$-th mergers in the original trellis (with full state number). An upper bound $d_{B}$ of the minimum distance in the case of the trellis designed with $h_{v}$, is computed by considering all the cases described in Eq. (28) i.e, using the difference sequences $\gamma$ in $\Gamma_{m}$ satisfying:

$$
\sum_{i=0}^{m-L} \gamma_{i}=\frac{2 \ell}{h_{v}}, \quad \ell \geq 0
$$

As $h_{v}=\frac{l_{v}}{p_{v}}$ with $l_{v}$ and $p_{v}$ being relatively prime integers and, as $\sum_{i=0}^{m-L} \gamma_{i}$ takes on integer values, Eq. (30) becomes

$$
\sum_{i=0}^{m-L} \gamma_{i}=2 \ell p_{v}, \quad \ell \geq 0 .
$$

2) Application to the Design of the Virtual Modulation Index: For a reduced-state receiver based upon $h_{v}$, the computation of the upper bound $d_{B}$ is as follows:

- First, we determine the set of difference sequences $\Gamma_{f}$ corresponding to the mergers of the first orders: they are identified from the reduced state trellis built upon $h_{v}$ (term (T2) in Eq. (22)). For a fixed $p_{v}$, we determine exhaustively the possible values of $m$ and their corresponding $\gamma$.

- We compute the normalized Euclidean distance $d(\gamma)$ given by Eq. (22) for every difference sequence found, since $\phi\left(t, \Delta_{h}, \gamma\right)+\phi\left(t, h_{v}, \gamma\right)=\phi(t, h, \gamma)$.

- The upper bound on the minimum distance $d_{B}$ is the lower bound of all of these computed normalized Euclidean distances $\left(d_{B}=\min _{\boldsymbol{\gamma} \in \Gamma_{f}} d(\gamma)\right)$.

We observe that the minimum value of $m$ for which Eq. (31) has solutions for $\ell \neq 0$ increases with $p_{v}$. Given $h, h_{v}$ will thus be chosen so that the value of $m$ yielding to the minimum distance in the MLSE trellis be inferior to the value of $m$ from which Eq. (31) has solution for $\ell \neq 0$. Among the set of $h_{v}$ satisfying this condition, we will choose the one which results in the best state number reduction.

Let us illustrate this principle with the CPM parameters used in Section III. The first two mergers are reported in Table II and an upper bound $d_{B}^{2}$ for the reduced state number trellis is plotted in Fig. 5 for several values of $h_{v}$. We observe that $d_{B}^{2}$ is the same for the reduced state number trellis and for the full state number trellis when $h_{v}=\frac{k}{5}$ with $k \wedge 5=1 . k \wedge p$ denotes the greatest common divisor of $k$ and $p$. Whereas if $h_{v}=\frac{k}{4}$, with $k \wedge 4=1$ there is a slight degradation when the transmission modulation index is different from $h_{v}$ and belongs to the interval $[0.51,0.56]$. For a detection with $h_{v}=\frac{k}{3}$ where $k \wedge 3=1$ there is also a degradation when $h \neq h_{v}$ belongs to the modulation index intervals $[0.34,0.44],[0.59,0.81],[0.9,1.13]$ or $[1.28,1.43]$. Finally, by using $h_{v}=\frac{k}{2}$ with $(k \wedge 2=1)$ we observe a degradation when $h \neq h_{v}$ is in the modulation index intervals [0.39, 0.67], $[0.87,1.191]$ or $[1.41,1.5]$. This is in accordance with the conclusions drawn from Fig. 3 and Fig. 4. We observed no error rate degradation in the case of $h=\frac{1}{4}$ and $h_{v} \in\left\{\frac{2}{3}, \frac{2}{5}\right\}$, whereas $h_{v}=\frac{2}{3}$ yielded a significant degradation in the case of $h=\frac{2}{5}$.

In order to check the validity of our approach, we added in Fig. 5 the values of the minimum distance in the reduced-state trellis, estimated through simulations based on the fact that the bit error probability $P_{e}$ for large $E_{b} / N_{0}$ is approximated by Eq. (17). For each value of $h$ and given a large $E_{b} / N_{0}$, we simulated the detection of the CPM with the proposed reduced-state trellis-based receiver to estimate the BER and to deduce an estimate of $d_{\mathrm{min}}^{2}(h)$ from the inverse function of $\mathrm{Q}(x)$ (the solution is unique since the function $\mathrm{Q}(x)$ is bijective). Note that we chose the value of $E_{b} / N_{0}$ to ensure a 
TABLE II: First two mergers in the reduced state number trellis for Quaternary 2RC

\begin{tabular}{|c|c|c|c|c|c|}
\hline & $\begin{array}{l}\text { MLSE } \\
h_{v}=h\end{array}$ & $\begin{array}{l}h_{v}=\frac{k}{5} \\
(k \wedge 5=1)\end{array}$ & $\begin{array}{l}h_{v}=\frac{k}{4} \\
(k \wedge 4=1)\end{array}$ & $\begin{array}{l}h_{v}=\frac{k}{3} \\
(k \wedge 3=1)\end{array}$ & $\begin{array}{l}h_{v}=\frac{k}{2} \\
(k \wedge 2=1)\end{array}$ \\
\hline $\begin{array}{l}\text { First } \\
\text { mergers }\end{array}$ & $\begin{array}{l}m=3 \\
\sum_{i=0}^{1} \gamma_{i}=0, \\
\gamma_{i} \in \Gamma_{3}\end{array}$ & $\begin{array}{l}m=3 \\
\sum_{i=0}^{1} \gamma_{i}=10 \\
\gamma_{i} \in \Gamma_{3}\end{array}$ & $\begin{array}{l}m=3 \\
\sum_{i=0}^{1} \gamma_{i}=8, \\
\gamma_{i} \in \Gamma_{3}\end{array}$ & $\begin{array}{l}m=2 \\
\gamma=(6,0)\end{array}$ & $\begin{array}{l}m=2 \\
\gamma=(4,0)\end{array}$ \\
\hline $\begin{array}{l}\text { Second } \\
\text { mergers }\end{array}$ & $\begin{array}{l}m=4 \\
\sum_{i=0}^{2} \gamma_{i}=0 \\
\gamma_{i} \in \Gamma_{5}\end{array}$ & $\begin{array}{l}m=4 \\
\sum_{i=0}^{2} \gamma_{i}=10 \\
\gamma_{i} \in \Gamma_{4}\end{array}$ & $\begin{array}{l}m=4 \\
\sum_{i=0}^{2} \gamma_{i}=8 \\
\gamma_{i} \in \Gamma_{4}\end{array}$ & $\begin{array}{l}m=3 \\
\sum_{i=0}^{2} \gamma_{i}=6, \\
\gamma_{i} \in \Gamma_{3}\end{array}$ & $\begin{array}{l}m=3 \\
\sum_{i=0}^{2} \gamma_{i}=4, \\
\gamma_{i} \in \Gamma_{3}\end{array}$ \\
\hline
\end{tabular}

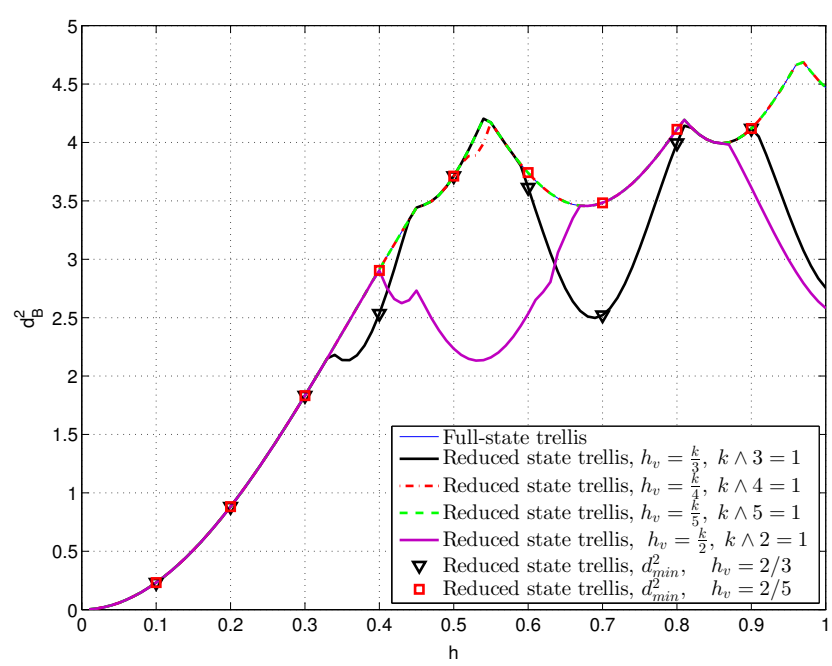

Fig. 5: Upper bound on the minimum distance as a function of $h$ in the reduced state number trellis for Quaternary 2RC CPM and different values of $h_{v}$

low BER, depending on the value of $h$ as follows: $E_{b} / N_{0}=16$ $\mathrm{dB}$ for $h \in\{0.1,0.2\}, E_{b} / N_{0}=12 \mathrm{~dB}$ for $h \in\{0.3,0.4\}$ and $E_{b} / N_{0}=10 \mathrm{~dB}$ for $h \in\{0.5,0.6,0.7,0.8,0.9\}$.

\section{B. Coded CPM: EXIT Charts Analysis}

In the case of uncoded CPM, the minimum distance is a good criterion to discard some values of the virtual reception modulation index. However, we have observed in Section III that given two different values of $h_{v}$, the performance of the two corresponding reduced-state receivers may coincide in the case of uncoded CPM and may differ in the case of BIC-CPM. Indeed, given a high enough signal to noise ratio, after the first iteration, the soft BCJR-based CPM detector of the BICCPM receiver yields the same decision as the hard Viterbibased CPM detector. The hard decision is given by applying a theshold on the log-likelihood ratio. Given two different values of $h_{v}$, the corresponding soft BCJR-based CPM detectors may deliver the same hard decisions but extrinsic information with highly different reliabilities.

The extrinsic information transfer (EXIT) chart is a powerful technique to analyze the behavior of iterative receivers [31]. In the case of BIC-CPM, the inner and the outer components of the receiver, namely the proposed CPM demodulator and the FEC decoder, exchange soft information on the encoded bits. The convergence behavior between these two modules can be studied by using EXIT chart analysis. The transfer characteristic curves can be obtained by considering the $a$ priori information delivered by the proposed CPM demodulator to the decoder [31]. In the following, we intend to analyze the influence of the choice of $h_{v}$ on the convergence of the reduced-state receiver from EXIT charts. Simulations are carried out with the Quaternary 2RC CPM as in previous sections.

In Fig. 6, we have considered $h=\frac{1}{4}$ and $E_{b} / N_{0}=1.5$ $\mathrm{dB}$. We have plotted the EXIT chart for the full-state receiver $\left(h_{v}=\frac{1}{4}\right)$ and for two reduced-state receivers $\left(h_{v}=\frac{2}{5}\right.$ and $h_{v}=\frac{2}{3}$ ). From Fig. 3, we have observed that the three receivers perform roughly the same for high codeword lengthes with a higher degradation for medium lengthes with $h_{v}=\frac{2}{3}$. From Fig. 6, we can also observe that for low-to-medium information values (beginning of the iterative process), the curves of the BCJR-based detectors seem parallel but shifted. The convergence threshold is thus better for $h_{v}=\frac{2}{5}$ than $h_{v}=$ $\frac{2}{3}$. The EXIT chart enables also to complete the complexity comparison by predicting the iteration number required to converge. Table III reports the complexity comparison in terms of trellis state number and minimum iteration number required to converge. We deduce that using $h_{v}=\frac{2}{5}$ at the reception allows to perform a good compromise between performance and complexity.

In Fig. 7 we have considered $h=\frac{2}{5}$ (same simulation conditions as for Fig. 4). The EXIT chart is plotted for the full-state receiver $\left(h_{v}=\frac{2}{5}\right)$ on the right and for the reducedstate receiver $\left(h_{v}=\frac{2}{3}\right)$ on the left. Different values of $E_{b} / N_{0}$ are simulated to compare the convergence thresholds. For $E_{b} / N_{0}=1.5 \mathrm{~dB}$, none of the receivers will be able to converge. For $E_{b} / N_{0}=2 \mathrm{~dB}$, only the full-state receiver will converge. For $E_{b} / N_{0}=2.5 \mathrm{~dB}$, both of them will converge. The difference between the respective thresholds of convergence is quite significative. The EXIT chart analysis is thus in accordance with the conclusions drawn from Fig. 4.

TABLE III: Trellis state number and predicted minimum iteration number for the reduced-state BIC-CPM receiver. Quaternary 2RC with $h=\frac{1}{4}$ for $\frac{E_{b}}{N_{0}}=1.5 \mathrm{~dB}$

\begin{tabular}{|c|c|c|}
\hline & $\begin{array}{c}\text { Trellis state } \\
\text { number }\end{array}$ & $\begin{array}{c}\text { Predicted minimum } \\
\text { iteration number }\end{array}$ \\
\hline$h_{v}=\frac{1}{4}$ & 32 & 4 \\
\hline$h_{v}=\frac{2}{5}$ & 20 & 5 \\
\hline$h_{v}=\frac{2}{3}$ & 12 & 9 \\
\hline
\end{tabular}




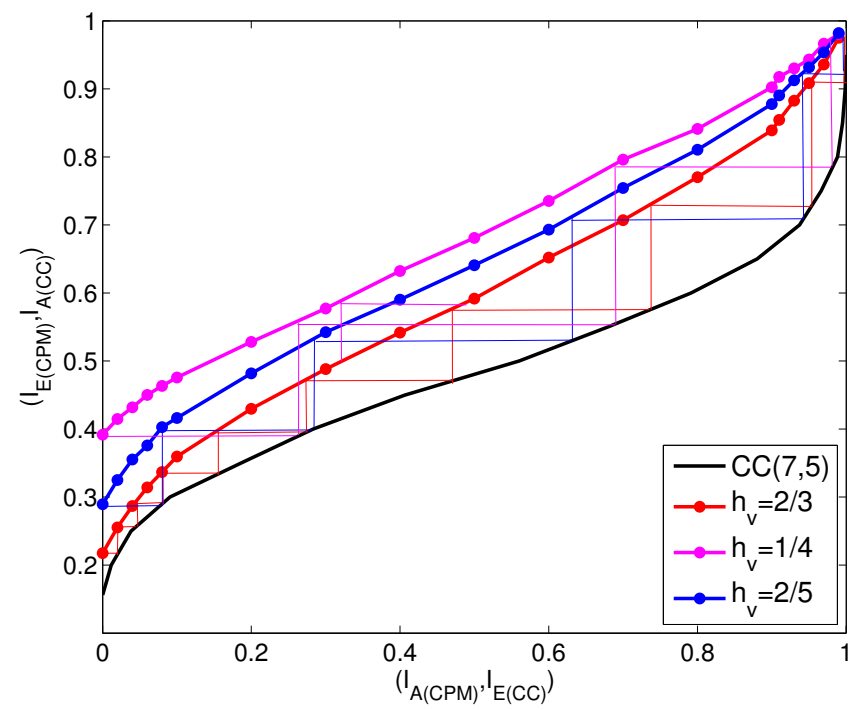

Fig. 6: EXIT chart for the reduced-state BIC-CPM receiver $\left(h_{v} \in\left\{\frac{1}{4}, \frac{2}{5}, \frac{2}{3}\right\}\right.$ ). Quaternary raised cosine CPM with $L=2$ and $h=\frac{1}{4}, \operatorname{CC}(7,5)$, pseudo-random interleaver, $\frac{E_{b}}{N_{0}}=1.5$ $\mathrm{dB}$.

\section{CONCLUSION}

This paper is focused on low complexity algorithms for CPM demodulation. The principle is to choose a modulation index to build the trellis at the receiver side which can be different from the modulation index of transmitted CPM waveform, hence enabling the detection of CPM waveforms with irrational modulation indices. Depending on the modulation index chosen to build the trellis for detection, the number of states can be reduced. Using modified branch metrics, both Viterbi algorithm for sequence detection and BCJR algorithm for soft-input soft-output CPM demodulation are derived. Even if a large range of modulation indices can be used at the receiver side, the complexity reduction (number of states in the trellis) is a first criterion to select a particular one. However, some modulation indices may degrade the error rate performance. We proposed in this paper two ways to discard such modulation indices. The first one is related to the uncoded CPM case (Viterbi detection) and is based on the minimal distance upper bound derivation, as a function of both the transmission modulation index and the modulation index used at the receiver side to design the trellis. The second one is related to the $\mathrm{BIC}-\mathrm{CPM}$ case, where an iterative receiver based on soft information exchange between the FEC decoder and the CPM detector is performed. In this case, avoiding values discarded from the minimal distance study, an EXIT chart analysis enables to discard the modulation indices which degrade the convergence of the iterative decoding algorithm. A
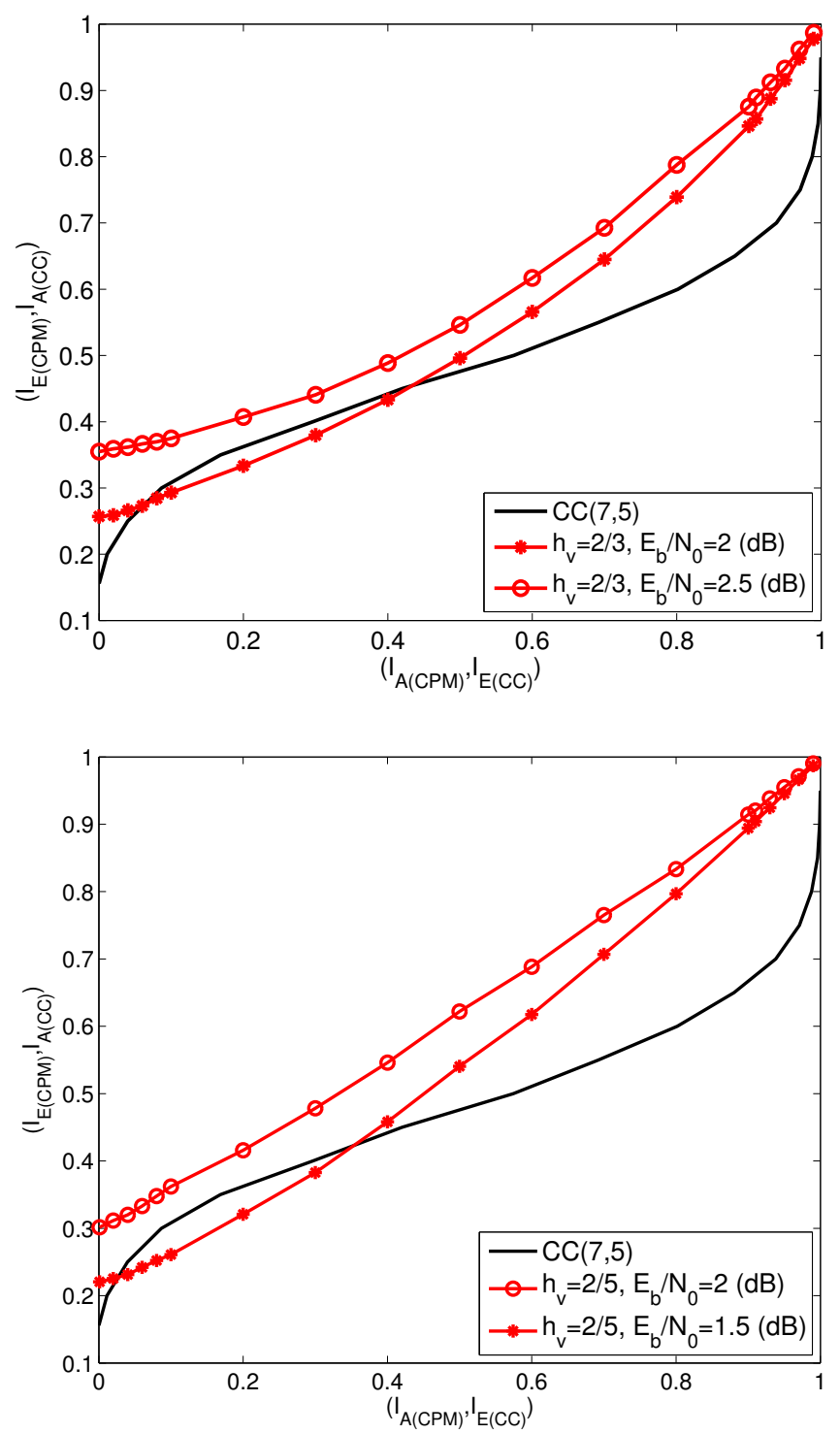

Fig. 7: EXIT chart for the BIC-CPM receiver. Quaternary raised cosine CPM with $L=2$ and $h=\frac{2}{5}$, CC(7,5), pseudorandom interleaver. Left side: reduced-state receiver, $h_{v}=\frac{2}{3}$, $\frac{E_{b}}{N_{0}} \in\{2,2.5\} \mathrm{dB}$. Right side: full-state receiver, $h_{v}=\frac{2}{5}$, $\frac{E_{b}}{N_{0}} \in\{1.5,2\} \mathrm{dB}$.

trade-off between the remaining possible modulation indices is finally possible by striking a balance between the convergence speed and the number of states in the trellis.

\section{REFERENCES}

[1] J. B. Anderson, T. Aulin, and C.-E. W. Sundberg, Digital Phase Modulation. New York: Plenum Press, 1986.

[2] C. H. Kuo and K. M. Chugg, "On the bandwidth efficiency of CPM signals," in Proc. IEEE Military Comm. Conf. (MILCOM), Oct.-Nov. 2004, pp. 218-224.

[3] G. E. Corazza (Ed.), Digital satellite communications. Springer, 2007.

[4] A. Graell i Amat, C. A. Nour, and C. Douillard, "Serially concatenated continuous phase modulation for satellite communications," IEEE Trans. Wireless Commun., vol. 8, pp. 3260-3269, Jun. 2009. 
[5] N. Noels and M. Moeneclaey, "Iterative multiuser detection of spectrally efficient fdma cpm,” Signal Processing, IEEE Transactions on, vol. 60, no. 10, pp. 5254-5267, Oct. 2012.

[6] M. K. Simon, Bandwidth-Efficient Digital Modulation With Applications to Deep-Space Communication. John Wiley \& Sons, 2003.

[7] Digital Video Broadcasting (DVB), "Second Generation DVB Interactive Satellite System; Part 2: Lower Layers for Satellite standard. DVB Document A155-2," Mar. 2011.

[8] T. Detwiler, S. Searcy, S. Ralph, and B. Basch, "Continuous phase modulation for fiber-optic links," Lightwave Technology, Journal of, vol. 29, no. 24, pp. 3659-3671, Dec. 2011.

[9] "IRIG Standard 106-04: Telemetry Standards, Group, Range Commanders Council, White Sands Missile Range, New Mexico, 2004," Available at http://www.ntia.doc.gov/legacy/osmhome/.

[10] Ericsson, "More than 50 billion connected devices," Tech. Rep., Feb. 2011.

[11] METIS, "Mobile and wireless communication enables for the twentytwenty information society," EU 7th Framework Programme project: http: / / www. metis2020.com.

[12] B. E. Rimoldi, "A decomposition approach to CPM," IEEE Trans. Inform. Theory, vol. 34, pp. 260-270, Mar. 1988.

[13] G. D. Forney, Jr., "The Viterbi algorithm," Proc. IEEE, vol. 61, pp. 268-278, Mar. 1973.

[14] T. Aulin and C.-E. W. Sundberg, "Continuous phase modulation-Part I: full response signaling," IEEE Trans. Commun., vol. 29, no. 3, pp. 196-209, Mar. 1981.

[15] T. Aulin, N. Rydbeck, and C.-E. W. Sundberg, "Continuous phase modulation-Part II: partial response signaling," IEEE Trans. Commun., vol. 29, no. 3, pp. 210-225, Mar. 1981.

[16] S. Simmons and P. Wittke, "Low complexity decoders for constant envelope digital modulations," IEEE Trans. Commun., vol. 30, pp. 1273 1280, Dec. 1983.

[17] A. Svensson, "Reduced state sequence detection of partial response continuous phase modulation," Communications, Speech and Vision, IEE Proceedings I, vol. 138, no. 4, pp. 256-268, 1991.

[18] A. Svensson, C.-E. Sundberg, and T. Aulin, "A class of reducedcomplexity Viterbi detectors for partial response continuous phase modulation," IEEE Trans. Commun., vol. 32, no. 10, pp. 1079-1087, 1984.

[19] J. Huber and W. Liu, "An alternative approach to reduced-complexity CPM-receivers," IEEE J. Select. Areas Commun., vol. 7, pp. 1437-1449, Dec. 1989.

[20] M. Costa, "A practical demodulator for continuous phase modulation," in Information Theory, 1994. Proceedings., 1994 IEEE International Symposium on, 1994, pp. 88-89.

[21] S. Simmons, "Simplified coherent detection of CPM," IEEE Trans. Commun., vol. 43, no. 234, pp. 726-728, 1995.

[22] W. Tang and E. Shwedyk, "A quasi-optimum receiver for continuous phase modulation," IEEE Trans. Commun., vol. 48, no. 7, pp. 1087 1090, 2000.

[23] P. A. Laurent, "Exact and approximate construction of digital phase modulations by superposition of amplitude modulated pulses (AMP)," IEEE Trans. Commun., vol. 34, pp. 150-160, Feb. 1986.

[24] G. K. Kaleh, "Simple coherent receivers for partial response continuous phase modulation," IEEE J. Select. Areas Commun., vol. 7, pp. 1427 1436, Dec. 1989.

[25] P. Moqvist and T. M. Aulin, "Serially concatenated continuous phase modulation with iterative decoding," IEEE Trans. Commun., vol. 49, pp. 1901-1915, Nov. 2001.

[26] N. Noels, M. Moeneclaey, F. Simoens, and D. Delaruelle, "A lowcomplexity iterative phase noise tracker for bit-interleaved coded CPM signals in AWGN,' IEEE Trans. Signal Processing, vol. 59, no. 9, pp. 4271-4285, Sep. 2011.

[27] A. Barbieri and G. Colavolpe, "Simplified soft-output detection of CPM signals over coherent and phase noise channels," IEEE Trans. Wireless Commun., vol. 6, no. 7, pp. 2486-2496, Jul. 2007.

[28] M. Messai, F. Guilloud, and K. Amis Cavalec, "A low complexity coherent CPM receiver with modulation index estimation," in Proc. European Signal Processing Conf., 2014.

[29] M. Messai, K. Amis Cavalec, and F. Guilloud, "A low complexity iterative soft detection for bit interleaved coded CPM," in ISTC 2014 : proceedings of the 8th International Symposium on Turbo Codes and Iterative Information Processing, 2014.

[30] L. R. Bahl, J. Cocke, F. Jelinek, and J. Raviv, "Optimal decoding of linear codes for minimizing symbol error rate," IEEE Trans. Inform. Theory, vol. 20, pp. 284-287, Mar. 1974.
[31] S. ten Brink, "Convergence behavior of iteratively decoded parallel concatenated codes," IEEE Trans. Commun., vol. 49, no. 10, pp. 1727 1737, Oct. 2001.

[32] R. Raheli, A. Polydoros, and C. Tzou, "Per-survivor processing: A general approach to MLSE in uncertain environments," IEEE Trans. Commun., vol. 43, pp. 354-364, February-April 1995.

[33] M. Figuera and J. Krogmeier, "Optimum and suboptimum receivers for M-ary CPM signals based upon the Laurent representation," in Vehicular Technology Conference, 2002. Proceedings. VTC 2002-Fall. 2002 IEEE 56th, vol. 4, 2002, pp. 2268-2272.

[34] U. Mengali and M. Morelli, "Decomposition of $M$-ary CPM signals into PAM waveforms," IEEE Trans. Inform. Theory, vol. 41, pp. 1265-1275, Sep. 1995

[35] G. Colavolpe and R. Raheli, "Reduced-complexity detection and phase synchronization of CPM signals," IEEE Trans. Commun., vol. 45, pp. 1070-1079, Sep. 1997

Malek MESSAI (S'14) was

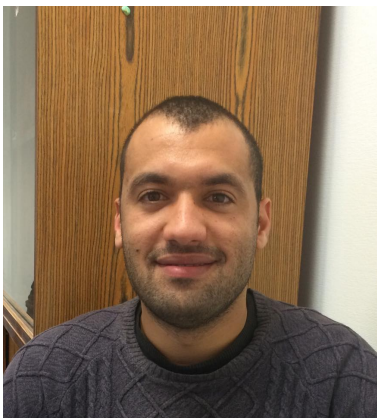
born in Jendouba, Tunisia in December 1988. He received the Dr. Eng. degree in Telecommunications Engineering in September 2012 from Higher School of Communication of Tunis (Sup'Com). Since October 2012, he has been pursuing the Ph.D. degree at the the Signal and Communication department of Telecom Bretagne, Brest, France. His main research interests include wireless communications, iterative decoding and spectrally efficient systems.

Karine Amis (M'00) received

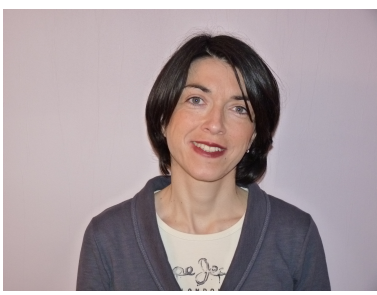
her Dipl.-Ing. degree from the Ecole Nationale Supérieure des Telecommunications de Bretagne (ENST Bretagne), France in 1998 and her $\mathrm{Ph} . \mathrm{D}$. degree from the University of Rennes 1, France in 2001. Since 2001, she is an associate professor at Telecom Bretagne, France. Her current research interests focus on communication and coding theory with application to MIMO systems, interference channels, cooperative communications and more recently power line communications.

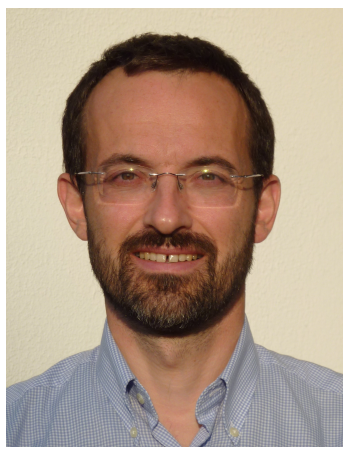

Frédéric Guilloud (S'02M'04) received the Eng. degree in Electronics from the Ecole Nationale Supérieure de l'Electronique et de ses Applications (ENSEA) in 1998, and is a former student of the Ecole Normale Supérieure de Cachan (ENS Cachan). He received the Ph.D. degree in 2004 from Telecom ParisTech in Signal and Communications, entitled "Generic Architecture for LDPC Codes Decoding". 
He is Professeur Agrégé in electronics and power electronics since 1999, and Associate Professor at Telecom Bretagne in the Signal and Communications department since 2004. His research and teaching activities are focused on error correcting codes and digital communications. 